\title{
Recursos Didácticos Comunicativos Y Audiovisuales Para La Enseñanza-Aprendizaje Del Idioma Inglés A Través De Aulas Virtuales
}

\author{
Armas Pesantez Paul Rolando \\ Magister en Lingüística Aplicada al Aprendizaje del Ingles \\ Docente de la Escuela Superior Politécnica de Chimborazo, Centro de \\ Idiomas, Ecuador \\ Armas Pesantez Washington Geovanny \\ Magister en Lingüística Aplicada al Aprendizaje del Ingles \\ Docente de la Universidad Nacional de Chimborazo, Ecuador

\section{Salazar Calderón Edison Hernán} \\ Magister en Lingüística Aplicada al Aprendizaje del Ingles \\ Docente de la Escuela Superior Politécnica de Chimborazo, Centro de \\ Idiomas, Ecuador

\section{Guadalupe Bravo Luis Oswaldo} \\ Magister en Lingüística Aplicada al Aprendizaje del Ingles \\ Docente de la Escuela Superior Politécnica de Chimborazo, Centro de \\ Idiomas, Ecuador \\ Orozco Yánez Gabriel Isaac \\ Ingeniero en Gestión Turística Y Hotelera \\ Docente de la Escuela Superior Politécnica de Chimborazo, Centro de \\ Idiomas, Ecuador
}

doi: 10.19044/esj.2017.v13n25p193 URL:http://dx.doi.org/10.19044/esj.2017.v13n25p193

\begin{abstract}
The purpose of the current research was the implementation of didactic audiovisual and communicative resources through a virtual classroom for the teaching-learning of English language, aimed to the first level students Languages School at Universidad Nacional de Chimborazo due to the lack of didactic material helping in the development of activities and tasks into the classroom. This fact impedes teachers and students reaching a higher level in the English Language teaching-learning process, this situation made necessary the implementation of resources adaptive to the pedagogical context and planned through a content manager or virtual classroom. The current research is quasi experimental, bibliographic, documental and descriptive which was applied to a sample where it was
\end{abstract}


necessary to consider an initial knowledge diagnose before applying the communicative and audiovisual resources, then it was necessary to carry out an evaluation at the end of it. It was also necessary to use a set of activities based on communicative and audiovisual resources framed within the micro curriculum guidelines with schedules and contents that were evaluated through questionnaires and a checklist. The instruments for collecting information allowed obtaining data in both pre-test and post-test. These qualifications were compared through a statistical test that allowed concluding that the use of the mentioned resources improved the English language teaching, at the same time it was possible to recommend its use within the curriculum for the First Level of the Languages Major.

Keywords: Communicative and audiovisual resources, Teaching Learning, English

\section{Resumen}

La presente investigación tuvo como objetivo la implementación de recursos comunicativos y audiovisuales didácticos a través de un aula virtual para la enseñanza-aprendizaje del idioma inglés, dirigido a los estudiantes de primer año de la escuela de idiomas de la Universidad Nacional de Chimborazo, debido a que no se cuenta con materiales didácticos que coadyuven al desarrollo de actividades y tareas en el aula, fenómeno que imposibilita a docentes y estudiantes mejorar en el proceso de enseñanza aprendizaje del idioma inglés, situación que hizo necesaria la implementación de recursos adaptativos a contextos pedagógicos planificados en a través de un gestor de contenidos o aula virtual. La presente investigación es cuasi experimental, bibliográfica, documental y descriptiva aplicada a una muestra, donde se consideró un diagnóstico inicial de conocimientos antes de aplicar los recursos comunicativos y audiovisuales, para luego realizar una evaluación al final del mismo. Se utilizó un conjunto de actividades basadas en los recursos comunicativos y audiovisuales enmarcados dentro de lineamientos de micro currículo con cronogramas y contenidos que fueron evaluadas mediante cuestionarios y una lista de cotejo. Los instrumentos de recolección de información permitieron obtener datos obtenidos en dos momentos: pre test y pos test, calificaciones que fueron comparadas a través de una prueba estadística, que permitió concluir que los usos de los recursos mencionados mejoraron la enseñanza aprendizaje del idioma inglés y a la vez se pudo recomendar su uso dentro del currículo del primer año de la carrera de Idiomas.

Palabras-Claves: Recursos Comunicativos y Audiovisuales, EnseñanzaAprendizaje, Inglés 


\section{Introducción}

En Ecuador, la enseñanza del idioma ingles a nivel de educación superior presenta falencias en los procesos de enseñanza aprendizaje pues no se ha seguido metodologías internacionales comprobadas que han dado resultados tales como English for Speakers of Other Languages (ESOL) Cambridge, de esta forma la tarea de enseñar está comprometida a desarrollar actividades contenidas en micro currículo (sílabos) que pueden ser sometidas a cambios o actualizadas cada vez que se presentan novedades o sugerencias a través de normativas curriculares generales o específicas en la carrera de idiomas. Sin duda el creciente desarrollo de pensamiento con metodologías, estrategias, la explosión del conocimiento conjuntamente con la tecnología y el incremento del cuerpo estudiantil y principalmente la exigencia colectiva de una educación más eficaz, presenta nuevos retos y paradigmas que pretenden influir positivamente en el proceso de enseñanza aprendizaje. Con base a lo anterior en el contexto de la presente investigación el docente debe desempeñar un papel gravitante en el mejoramiento de la educación, utilizando recursos comunicativos y audiovisuales a través de recursos actualizados a través de plataformas virtuales donde se puede organizar, planificar, poner a disposición del estudiante recursos y actividades y hacer un seguimiento a través de evaluaciones. Por tal motivo la presente investigación, ofrece nuevos recursos que contribuyen a los estudiantes y docentes en el aprendizaje y enseñanza del idioma Inglés a través de diferentes materiales como recursos comunicativos y audiovisuales.

Con la implementación de las actividades en un aula virtual con recursos comunicativos audiovisuales, pretendió evitar que los docentes y estudiantes sigan utilizando recursos tradicionales, pues uno de los objetivos fue poner a disposición de los actores de la investigación recursos intangibles, para obtener un mayor grado de aprendizaje estudiantil, ya que el mayor grado de conocimiento se adquiere por medio de los sentidos humanos con instrumentos que motiven cognitivamente a través de la tecnología como son la vista y el oído.

La inclusión de la tecnología en el ámbito educativo incluye fortalecer las destrezas en el manejo de las mismas por parte de sus actores, para alcanzar la evolución y una mejor enseñanza-aprendizaje del idioma inglés, por tal motivo este proyecto revela la importancia de contribuir con recursos tecnológicos didácticos como la guía basada en técnicas para el uso de videos y canciones que estimularán a los estudiantes del primer año de la Escuela de Idiomas de la Universidad Nacional de Chimborazo, enrumbándolos en el camino correcto del aprendizaje del idioma Inglés.

Involucrando la investigación a estudios previos sobre el factor negativo del cual imposibilita el progreso de la enseñanza del idioma inglés, 
se encontró referencias que señalan que "la enseñanza del Inglés en los sistemas de educación superior ha sufrido varios cambios. Como toda innovación propuesta por unos pocos y puesta en marcha por un grupo mayor, estos cambios curriculares han sido recibidos de distintas maneras por los variados agentes involucrados en la enseñanza del inglés como lengua extranjera". (FARIAS, 2000).

La importancia de la presente investigación es contribuir con la necesidad de aprender el idioma inglés que ha alcanzado a nivel mundial diferentes ámbitos incluido el educativo; a nivel superior la enseñanzaaprendizaje del idioma inglés, radica en el efecto de ser una herramienta para consolidar un proceso real de enseñanza-aprendizaje, más aún si se lo considera como una futura profesión; como es el caso de los estudiantes de la Escuela de Idiomas, mismos que al culminar con su intrusión superior, tendrán como fin el poderse desenvolver de la mejor manera ya como profesional en la enseñanza del idioma (licenciado en idiomas) en cualquier nivel en el que su servicio sea requerido, mostrando la importancia del idioma en el aspecto académico e incluso contribuyendo con el desarrollo personal de los estudiantes en su vida estudiantil y profesional.

Por los antecedentes antes mencionados se hace necesario que se implemente técnicas para el uso de videos y canciones como recursos comunicativos y audiovisuales para mejorar la enseñanza - aprendizaje del idioma inglés, para los estudiantes de primer año de la escuela de Idiomas de la UNACH.

Para llegar a la meta de la presente investigación se consideró en primera instancia identificar qué tipo de material audiovisual se puede utilizar en los procesos de enseñanza-aprendizaje del idioma Inglés, para luego realizar el diseño de una guía basada en técnicas para la adecuada utilización de videos y canciones en el proceso de enseñanza - aprendizaje del idioma inglés. La guía consta de un DVD con actividades interactivas participativas que orientan al docente y el estudiante en el desarrollo de cada una, acompañada de una rúbrica de evaluación y lista de cotejo, que permite la evaluación y recolección de datos que son tabulados, analizados, interpretados y utilizados para comprobar su incidencia en los estudiantes del primer año de Idiomas de la Universidad Nacional de Chimborazo.

\section{Metodología}

La investigación aplicó un diseño cuasi experimental, ya que se aplicó a un grupos de estudiantes del primer año de la carrera de inglés. El mismo grupo fue evaluado antes (diagnóstico) y después (evaluación final). Al inicio se realizó un diagnóstico de conocimientos contenidos en el silabo y representan a los estudiantes antes de aplicar la técnica con el uso de videos y canciones como recursos comunicativos y audiovisuales. 
Posteriormente con el mismo grupo de estudiantes, se desarrollaron las actividades de forma planificada y sistemática implementadas en el aula virtual que también fueron evaluadas para obtener calificaciones que permitan determinar si se incidió en el aprendizaje, es decir establecer las relaciones de causalidad entre el objeto de estudio y sus elementos constituyentes o el efecto de la variable independiente sobre la dependiente.

Los tipos de investigación que se utilizó fueron: científica ya que se recogió información relevante la misma que fue verificada y aplicada al conocimiento. De campo, ya que se interpretó el contexto de la investigación y se propuso una solución al problema; además se trabajó en un ambiente donde población y la muestra cumplen el papel de ser la fuente de datos para ser analizados. Cuantitativa ya que se evaluó la muestra en los escenarios a través de una rúbrica que permitió obtener valores cuantificables para ser comparados mediante una prueba estadística. Descriptiva pues se describe el fenómeno tal como aparecen en la realidad en un momento determinado. Y finalmente bibliográfica que fundamentó el conocimiento de los contenidos de la guía como técnica a implementarse.

Los métodos de la investigación fueron: factual, puesto que los datos obtenidos son de las pruebas acertadas, que fueron contrastados con hechos reales para poner a prueba una hipótesis. Analítico ya que se estudió por separado cada una de las variables para ver las relaciones existentes entre las mismas. Inductivo - deductivo porque se partió de un ámbito general a uno particular.

Se realizó la observación de los participantes sujetos del problema, a fin de evidenciar mediante listas de cotejo el desempeño del aprendizaje. Se utilizó también una rúbrica de evaluación que fue validada por profesionales pares del área de inglés. La rúbrica fue contextualizada e incluía preguntas precisas para evaluar los indicadores como elementos para la demostración de la hipótesis.

Los instrumentos de recolección de información fueron puestos en consideración de pares académicos para que sean verificados y validados. Se consideró la elaboración de las matrices de operacionalización de variables, para estructurar los cuestionarios. Para realizar la recolección de la información, se planificó y programó un conjunto de actividades de forma sistemática distribuidas como actividades y recursos previstos en el aula virtual. Para la revisión crítica de la información formulada a través del instrumento de esta investigación, se utilizó la tabulación, análisis e interpretación de resultados.

La población representa los 33 estudiantes y 1 docente que representa el grupo experimental del primer año de la carrera de inglés de la Universidad Nacional de Chimborazo, y se tomó la totalidad de la población para diagnosticarla (Pre test) y evaluarla al final (Post test) de haber aplicado 
los recursos comunicativos y audiovisuales en el proceso de enseñanza aprendizaje del idioma inglés. Es decir, la muestra fue evaluada en dos momentos antes y después de haber aplicado la técnica para la enseñanza aprendizaje de idioma inglés.

Los datos recolectados de la investigación, fueron analizados y sometidos a una prueba estadística, identificando tendencias coyunturales a los objetivos y la hipótesis. La comprobación de hipótesis se lo hizo a través de una prueba estadística, con el asesoramiento de un especialista del área, cuyos resultados permitirán establecer objetivamente conclusiones y recomendaciones de la investigación.

En primera instancia se identificó y definió con base a recursos comunicativos y audiovisuales las actividades que estén acordes a los sílabos del primer nivel de la carrera de inglés; entre las que se consideró el video, lluvia de ideas, role play y juegos; así también While viewing and Active viewing, Segment viewing, Silent viewing. Luego se implementó un aula virtual alojada en servidores de gestores de contenidos en la Universidad Nacional de Chimborazo, que permitió alojar las publicaciones a través de actividades, incluyendo objetos de aprendizaje. Posteriormente a través de actividades publicadas en el aula virtual, se desarrolló el proceso de enseñanza aprendizaje del idioma inglés en los estudiantes de primer nivel de forma planificada y sistemática. Fueron planteadas 20 actividades acordes a los contenidos del sílabo de la carrera, desarrolladas durante 4 meses. Cada actividad fue evaluada mediante listas de cotejo que permitió obtener una calificación. Luego se compararon las calificaciones obtenidas en los escenarios del antes y después para determinar si el proceso de enseñanza aprendizaje del idioma inglés fue mejorado.

\section{Resultados}

A continuación, se presentan los resultados finales obtenidos por 33 estudiantes del primer año de la carrera de Idiomas de la Universidad Nacional de Chimborazo. Los puntajes son el resultado de un cuestionario antes y después de aplicar la técnica y que permitió evaluar el aprendizaje en el contexto de la investigación. Las calificaciones finales se representan en la siguiente tabla. 


\begin{tabular}{|c|c|c|c|}
\hline \multirow[b]{2}{*}{ Tabla 1: } & \multirow[b]{2}{*}{ Calificaciones pre test vs post test de los 33 estudiantes } & \multicolumn{2}{|c|}{ Calificación } \\
\hline & & Pre TEST & Post TEST \\
\hline & $\begin{array}{l}\text { Estudiante } 1 \\
\end{array}$ & 2,7 & 5,0 \\
\hline & Estudiante 2 & 5,1 & 4,1 \\
\hline & Estudiante 3 & 6,2 & 5,3 \\
\hline & Estudiante 4 & 2,0 & 5,0 \\
\hline & Estudiante 5 & $\overline{7,0}$ & 5,0 \\
\hline & Estudiante 6 & 2,0 & 2,2 \\
\hline & Estudiante 7 & 3,0 & 6,0 \\
\hline & Estudiante 8 & 3,5 & 7,2 \\
\hline & Estudiante 9 & 1,1 & 6,7 \\
\hline & Estudiante 10 & 2,0 & 5,5 \\
\hline & Estudiante 11 & 4,0 & 7,5 \\
\hline & Estudiante 12 & 6,5 & 7,2 \\
\hline & Estudiante 13 & 1,5 & 5 \\
\hline & Estudiante 14 & 3,0 & 6,0 \\
\hline & Estudiante 15 & 2,7 & 5,5 \\
\hline & Estudiante 16 & 2,0 & 3,2 \\
\hline & Estudiante 17 & 1,5 & 5,2 \\
\hline & Estudiante 18 & 3,5 & 6,1 \\
\hline & Estudiante 19 & 4,5 & 6,1 \\
\hline & Estudiante 20 & 3,2 & 5,1 \\
\hline & Estudiante 21 & 7,2 & 8,2 \\
\hline & Estudiante 22 & 5,3 & 6,2 \\
\hline & Estudiante 23 & 2,3 & 3,9 \\
\hline & Estudiante 24 & 1,5 & 5,4 \\
\hline & Estudiante 25 & 6,5 & 7,1 \\
\hline & Estudiante 26 & 5,0 & 5,0 \\
\hline & Estudiante 27 & 4,8 & 6,1 \\
\hline & Estudiante 28 & 5,6 & 8,0 \\
\hline & Estudiante 29 & 4,8 & 7,6 \\
\hline & Estudiante 30 & 2,3 & 6,2 \\
\hline & Estudiante 31 & 2,7 & 5,0 \\
\hline & Estudiante 32 & 5,1 & 4,1 \\
\hline & Estudiante 33 & 6,2 & 5,3 \\
\hline
\end{tabular}

Fuente: Evaluaciones pre-test y post test de los estudiantes del primer nivel de la carrera de Ingles de la UNACH

Elaborado por: Armas Paul, 2015

\section{PRE TEST VS POST TEST}

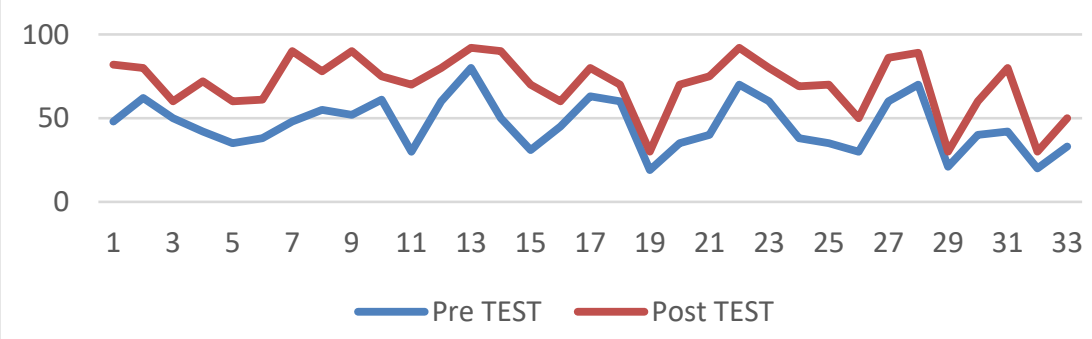

Gráfico 1: Pre test vs Post test

Fuente: Evaluaciones pre-test y post test 
En la siguiente tabla se presenta el descriptivo de los escenarios, donde la media del Pre test es menor que la media del Post test.

Tabla 2: Descriptivos de la prueba estadística

\begin{tabular}{|c|c|c|c|c|}
\hline \multicolumn{5}{|c|}{ Descriptivos } \\
\hline & & & Estadístico & Error estándar \\
\hline \multirow[t]{13}{*}{ PRE TEST } & \multicolumn{2}{|l|}{ Media } & 3,806 & ,3106 \\
\hline & \multirow{2}{*}{$\begin{array}{c}95 \% \text { de intervalo de } \\
\text { confianza para la media }\end{array}$} & Límite inferior & 3,173 & \\
\hline & & Límite superior & 4,439 & \\
\hline & \multicolumn{2}{|c|}{ Media recortada al 5\% } & 3,763 & \\
\hline & \multicolumn{2}{|c|}{ Mediana } & 3,500 & \\
\hline & \multicolumn{2}{|c|}{ Varianza } & 3,184 & \\
\hline & \multicolumn{2}{|c|}{ Desviación estándar } & 1,7843 & \\
\hline & \multicolumn{2}{|c|}{ Mínimo } & 1,1 & \\
\hline & \multicolumn{2}{|c|}{ Máximo } & 7,2 & \\
\hline & \multicolumn{2}{|c|}{ Rango } & 6,1 & \\
\hline & \multicolumn{2}{|c|}{ Rango intercuartil } & 3,0 & \\
\hline & \multicolumn{2}{|c|}{ Asimetría } & ,312 & ,409 \\
\hline & \multicolumn{2}{|c|}{ Curtosis } & $-1,072$ & ,798 \\
\hline \multirow[t]{13}{*}{ POST TEST } & \multicolumn{2}{|c|}{ Media } & 5,876 & ,2375 \\
\hline & \multirow{2}{*}{$\begin{array}{l}95 \% \text { de intervalo de } \\
\text { confianza para la media }\end{array}$} & Límite inferior & 5,392 & \\
\hline & & Límite superior & 6,360 & \\
\hline & \multicolumn{2}{|c|}{ Media recortada al $5 \%$} & 5,933 & \\
\hline & \multicolumn{2}{|c|}{ Mediana } & 6,000 & \\
\hline & \multicolumn{2}{|c|}{ Varianza } & 1,862 & \\
\hline & \multicolumn{2}{|c|}{ Desviación estándar } & 1,3645 & \\
\hline & \multicolumn{2}{|c|}{ Mínimo } & 2,2 & \\
\hline & \multicolumn{2}{|c|}{ Máximo } & 8,2 & \\
\hline & \multicolumn{2}{|c|}{ Rango } & 6,0 & \\
\hline & \multicolumn{2}{|c|}{ Rango intercuartil } & 2,1 & \\
\hline & \multicolumn{2}{|c|}{ Asimetría } &,- 555 & ,409 \\
\hline & \multicolumn{2}{|c|}{ Curtosis } & ,436 & ,798 \\
\hline
\end{tabular}

Fuente: SPSS

Elaborado por: Armas Paul, 2015

EPost Test $>$ EPre test

$5,876>3,806$

Es decir que los recursos comunicativos y audiovisuales en un entorno interactivo como un aula virtual motivan la atención de los estudiantes, mostrando mayor interés, concentración e interactividad a través de las actividades que permitieron mejorar la enseñanza del aprendizaje del idioma inglés.

\section{Prueba de normalidad}

La muestra representa 33 estudiantes por lo que utilizaremos la prueba de Shapiro-Wilk 
Tabla 3: Pruebas de normalidad

\begin{tabular}{|c|c|c|c|c|c|c|}
\hline & \multicolumn{3}{|c|}{ Kolmogorov-Smirnov $^{\mathrm{a}}$} & \multicolumn{3}{c|}{ Shapiro-Wilk } \\
\cline { 2 - 7 } & Estadístico & Gl & Sig. & Estadístico & gl & Sig. \\
\hline PRE TEST &, 118 & 33 &, $200^{*}$ &, 944 & 33 &, 091 \\
POST TEST &, 139 & 33 &, 104 &, 961 & 33 & 268 \\
\hline
\end{tabular}

*. Esto es un límite inferior de la significación verdadera.

a. Corrección de significación de Lilliefors

Fuente: SPSS

Elaborado por: Armas Paul, 2015

Tabla 4: Análisis de prueba de Normalidad:
Pre Test
P-valor $=0.091$
$>$
$\alpha=0.05$
Post test P-valor $=0.268$
$\alpha=0.05$

CONCLUSIÓN: El nivel de significancia obtenido tanto en el pre y post test cumple con la prueba de normalidad; es decir P - valor $>\alpha$ por lo que se aceptar H1

Fuente: SPSS

Elaborado por: Armas Paul, 2015

Debido a que el nivel de significancia del POST TEST y del POST TES es mayor que alpha, se acepta $\mathrm{H} 1$, es decir que los datos provienen de una distribución normal y se procede a aplicar t-student.

Tabla 5: Estadísticos de muestras relacionadas

\begin{tabular}{|cc|c|c|c|c|}
\hline & Media & $\mathrm{N}$ & $\begin{array}{c}\text { Desviación } \\
\text { estándar }\end{array}$ & $\begin{array}{c}\text { Media de error } \\
\text { estándar }\end{array}$ \\
\hline \multirow{2}{*}{ Par 1 } & PRE TEST & 3,806 & 33 & 1,7843 &, 3106 \\
& POST TEST & 5,876 & 33 & 1,3645 &, 2375 \\
\hline
\end{tabular}

Como se puede observar la media del Post test es mayor que la media del Pre Test

Tabla 6: Prueba de muestras emparejadas

\begin{tabular}{|c|c|c|c|c|c|c|c|c|}
\hline & \multicolumn{5}{|c|}{ Diferencias emparejadas } & \multirow[b]{3}{*}{$\mathrm{t}$} & \multirow[b]{3}{*}{ gl } & \multirow{3}{*}{$\begin{array}{c}\text { Sig. } \\
\text { (bilateral) }\end{array}$} \\
\hline & \multirow[b]{2}{*}{ Media } & \multirow{2}{*}{$\begin{array}{c}\text { Desviación } \\
\text { estándar }\end{array}$} & \multirow{2}{*}{$\begin{array}{c}\text { Media de } \\
\text { error } \\
\text { estándar }\end{array}$} & \multicolumn{2}{|c|}{$\begin{array}{c}95 \% \text { de intervalo de } \\
\text { confianza de la } \\
\text { diferencia } \\
\end{array}$} & & & \\
\hline & & & & Inferior & Superior & & & \\
\hline $\begin{array}{cc}\text { Par PRE TEST } \\
1 & \text { - POST } \\
& \text { TEST }\end{array}$ & $-2,0697$ & 1,6745 & ,2915 & $-2,6635$ & $-1,4759$ & $7, \overline{100}$ & 32 & ,000 \\
\hline
\end{tabular}

P-valor $=0.000$

$<\quad \alpha=0.05$

CONCLUSIÓN: En la prueba de las muestras relacionadas se puede observar que el nivel de significancia es de cero por tanto menor que alpha; por lo que se rechaza la Ho y se acepta H1. 
Es decir que los recursos comunicativos y audio visual a través de un aula virtual mejoraron la enseñanza aprendizaje del idioma inglés en los estudiantes del primer año de la Universidad Nacional de Chimborazo

\section{Discusión}

Se debe resaltar que la influencia de recursos que permiten interactuar en el proceso de enseñanza aprendizaje a través de un aula virtual, deben ser dirigidos por el docente mediante una guía en el desarrollo de las actividades. "Con toda probabilidad es casi imposible que un estudiante aprenda en una lengua extraña lo que no sabe hacer en la suya". (López, 1985).

La inclusión de los recursos tecnológicos en la comunicación y en la enseñanza han contribuido a la evolución del trabajo colaborativo, donde los principios didácticos que subyacen son la interactividad, flexibilidad e individualización de la enseñanza. En el contexto de la presente investigación existe el acompañamiento del docente en el desarrollo de las actividades soportadas con recursos comunicativos y audiovisuales, y que pretenden contribuir con la consecución de dos grandes objetivos: la mejora de la calidad del aprendizaje (por ejemplo trazando programas e itinerarios individualizados) y la accesibilidad, por parte de un mayor porcentaje de población, a la educación y la formación (Díez de la Cortina, 2005)

La metodología concebida como un método (que se adopta para enseñar o educar) o conjunto de métodos que el docente emplea para facilitar y efectivizar su labor como mediador del conocimiento en el proceso de enseñanza-aprendizaje en el idioma inglés, pueden ser adoptados con la aplicación de técnicas que contienen instrumentos como recursos comunicativos y audiovisuales organizados bajo normas micro curriculares, que permiten explorar experiencias que pueden ser aplicadas para obtener resultados favorables en los proceso de enseñanza aprendizaje que fueron aplicados en la presente investigación obteniendo importantes datos para encontrar la correlación entre variables dependientes e independientes.

La metodología que el docente emplee en la enseñanza del idioma inglés que esta normada por un silabo debe periódicamente reformularse para poder incluir resultados de nuevas investigaciones que aporten al nivel cognoscitivo y evolutivo de los estudiantes, teniendo en cuenta que la enseñanza aprendizaje de un nuevo lenguaje (sistema para la comunicación humana, el mismo que es usado para dar y recibir información), el cual en su esencia está compuesto por: sonidos, sintaxis, fonología y semántica se lo debe desarrollar por medio del dominio de las destrezas que este posee, particularmente en nuestro caso las destrezas del idioma inglés.

Hoy en día, la enseñanza está influenciada por la implementación de diferentes metodologías y sin duda por los avances tecnológicos (recursos 
audio-visuales), mismos que a través de su correcta aplicación y uso en el desarrollo de las actividades en el aula o laboratorio tecnológico, puede brindar mayores y mejores resultados en el transcurso y al final del proceso de enseñanza-aprendizaje, considerando que es imprescindible y esencial que en tanto el docente y estudiante estén familiarizados con los avances tecnológicos (recursos audio-visuales) y la metodología para ponerlos en uso durante sus actividades a través de técnicas claramente definidas.

En el sistema educativo a nivel superior la enseñanza aprendizaje del idioma inglés es un requisito para la graduación de pregrado y requisito fundamental a nivel de postgrado, convirtiendo en una necesidad que ha sido adoptada y que ha sido impactada por investigaciones para mejorar a través de metodologías y estrategias para la formación de los estudiantes del primer año de la carrera de inglés.

Sin duda, un factor determinante para que los recursos tengan mejores resultados es la implementación de programas intensivos de enseñanza del idioma inglés a nivel universitario, ya que en EEUU normalmente requieren veinte horas o más por semana, y en Ecuador a penas se estudian 4 horas semanales durante algunos casos hasta 6 semestres que generalmente no representan ni favorecen el aprendizaje. Y sin duda es imprescindible el uso de tecnología, dentro de la que encontramos diferentes reproductores de audio y video (medios audio-visuales), de tal manera que los utilicemos a todos ellos con facilidad y podamos conseguir mejores resultados tanto para maestros como para los alumnos de manera principal en dicho proceso.

Fue fundamental en la presente investigación que los recursos sean aplicados como recursos comunicativos y audiovisuales a través de las actividades sean relacionadas al micro currículo para orientar el aprendizaje al nivel académico de los estudiantes del primer año de la Universidad Nacional de Chimborazo.

Los recursos tecnológicos sin duda recursos complementarios que se pueden utilizar en el proceso educativo, por ser poseedores de características de interacción, actualidad, finalidad, utilidad, adecuación e interés, de esta manera contribuyen a la proyección de material didáctico y facilitan el entendimiento de varios temas por medio de un enfoque visual y auditivo que motiven al estudiante a aprender con mucho más interés que con las formas tradicionales de escuchar y repetir de manera interminable lo que dice el maestro.

Los recursos comunicativos y audiovisuales proyectadas con la ayuda de los recursos tecnológicos contribuyen como nuevas formas de enseñanza de vocabulario y proveen un lenguaje real, mientras que el video permite a los estudiantes ver las expresiones faciales, el lenguaje del cuerpo, al mismo tiempo que ellos oyen la pronunciación, la entonación, el acento, y el ritmo 
del lenguaje. Además, los videos y canciones presentan el idioma real hablado a una velocidad normal y con acento genuino, en este punto es importante aclarar que el profesor debe tomar en cuenta estos últimos factores mencionados para convertirlos en una ventaja de aprendizaje para los estudiantes al diseñar un plan de clase. Es decir que la ventaja de usar adecuadamente los videos (películas) en la enseñanza del inglés radica en que estos nos transmiten directamente los contenidos de un idioma, la información real de una cultura diferente a través de imágenes y sonidos.

Según Kennedy (1984), consideran que la "información en su forma original (authentic materials) es el material a partir del cual el maestro trabaja (el mismo que lo podemos encontrar plasmados en los videos), para conseguir los fines lingüísticos propuestos, esto con miras a el adiestramiento y elevación del nivel lingüístico de los estudiantes hasta un punto que les permita enfrentarse con éxito a los textos de diversa naturaleza" que en el ejercicio de su profesión utilizarán la lengua inglesa como vehículo de difusión de sus conocimientos y la promulgación del mismo idioma. La técnica o técnicas que se utilizará en la enseñanza del idioma inglés deben ser escogidas detenidamente y teniendo en cuenta siempre las necesidades que los estudiantes tienen, es así que (Gómez, 1995) sugiere a los maestros de inglés el uso de los pasos esenciales para diseñar una lección. Los docentes pueden usar el video como una actividad en tres pasos: Pre-viewing, while-viewing y Post-viewing activity, en cada uno de ellos, el docente puede incluir una o varias técnicas según lo considera necesario, no se debe olvidar que el diseño de planes de clases donde la variedad marca la pauta depende de cada docente.

Como resultado de la aplicación de los recursos comunicativos y audiovisuales al contexto de la presente investigación, se pudo obtener las evaluaciones a través del seguimiento de las actividades participativas y planificadas, mediante reuniones periódicas de trabajo en clase entre los personajes involucrados, lo cual fue de utilidad y sirvió como aporte para la elaboración del informe final del Proyecto.

Así también se realizó una demostración de la utilización de la guía didáctica (Learning English Through Videos and Songs) con los estudiantes de primer año de la Escuela de Idiomas de la Universidad Nacional de Chimborazo; los mismos que se sintieron motivados y expresaron que el uso de este recurso es una buena alternativa para el correcto aprendizaje del Idioma Inglés, así también señalaron que el uso que se le dé a este recurso debe ser continuo.

\section{Conclusion}

La identificación del tipo de material audiovisual como recurso comunicativo para el proceso de enseñanza-aprendizaje del idioma Inglés 
permitió determinar la línea base para la elaboración de las actividades a desarrollarse.

La identificación de los recursos comunicativos y audiovisuales permitió diseñar y publicar actividades en un aula virtual de forma planificada y sistemática con la finalidad de que los contenidos se desarrollen y se apliquen para obtener información que fue utilizada para conocer su incidencia y comprobación de la hipótesis.

Como instrumento para el docente y el estudiante se elaboró un aula virtual que facilitó el desarrollo de las actividades, las mismas que fueron evaluadas y que sirvió en la recolección de la información a través de una rúbrica de evaluación.

En la prueba de muestras relacionadas mediante la prueba estadística de t-student, el nivel de significación es de 0,000 es menor a alpha, condición que permitió rechazar la hipótesis nula y afirmar que la aplicación de la técnica del uso de video como recurso comunicativo y audio visual mejoró la enseñanza aprendizaje del idioma inglés en los estudiantes del primer año de la Universidad Nacional de Chimborazo

\section{Recomendaciones}

Las actividades incluidas en la guía pueden ser adaptadas a diferentes niveles de enseñanza de inglés atendiendo los contextos para su aplicación las mismas que pueden ser incrementadas en número que implique la participación de los estudiantes y docentes a fin de mejorar los procesos de enseñanza aprendizaje del idioma inglés.

Se recomienda que las actividades contenidas en el aula virtual sean inducidas a fin de despertar mayor interés en el desarrollo de las mismas, por lo que se podrá obtener nuevos resultados en el mejoramiento del aprendizaje y enseñanza del idioma inglés.

\section{References:}

1. Bernárdez Mariano L. (2007) "DISEÑO, PRODUCCIÓN E IMPLEMENTACIÓN DE E-LEARNING" Ed. AuthorHouse, USA

2. Brutt-Griffler, J. (2002). World English: A Study of Its Development. Multilingual Matters.

3. Cabañas Valdiviezo, J.; Ojeda Fernández, Yesenia (2003) "AULAS VIRTUALES COMO HERRAMIENTA DE APOYO EN LA EDUCACIÓN DE LA UNIVERSIDAD NACIONAL MAYOR DE SAN MARCOS" Tesis SISBIB Lima Perú

4. Dr. OCHOA, Marcelo, técnicas de estudio novena edición. EDIPCENTRO. Riobamba G1999. 
5. ESCUDERO ( 2003) PROYECTO FACTIBLE, Elaboración de Material Didáctico para la Enseñanza del Inglés dirigido a los niños de 6 a 8 años. Escuela de Idiomas.

6. García Teske, E. (2007). "Los discursos sobre las nuevas tecnologías en contextos educativos: ¿Qué hay de nuevo en las nuevas tecnologías?", en Revista Iberoamericana de Educación. ISSN: 16815653) $\mathrm{N}^{\circ} 41 / 4$. OEI

7. Griffee, D.T. (1992). Songs in Action. London: Prentice Hall.

8. Gvirtz, S. y Necuzzi, C. (2011) "Entrevista a Mariona Grané" en Educación y tecnología. Las voces de los expertos. Bs As, Anses,

9. Kennedy, G. (1998). An introduction to corpus linguistics. London: Longman

10. Loayza Álvarez, Roger (2002) "FACILITACIÓN Y CAPACITACIÓN VIRTUAL EN AMÉRICA LATINA", Colombia.

11. López, F. (1985), Teoría de la conducta México: Trillas

12. $M^{\mathrm{a}}$ Mar Bermejo del Campo. Maestra de Inglés. Artículo en la revista "Escuela en Acción. Infantil" Mayo 2004

13. Ms. PEZO, 1999, Elsa, didáctica general. CODEU. Quito

14. NERICI (1973) INTRODUCCIÓN HACIA UINA DIDÁCTICA GENERAL DINAMICA, Editorial K APELUSZ, SA, Buenos Aires.

15. Piaget, J. (1965). El lenguaje y el pensamiento. Buenos Aires: $\mathrm{CH}$

16. TORRES, Mónica (2004) DIDACTIC to Children

17. Salinas, J (2004). "Innovación docente y uso de las TIC en la enseñanza universitaria" en Revista de Universidad y Sociedad del Conocimiento. UOC. Vol.1-N ${ }^{\circ} 1$ : http://www.uoc.edu/rusc/dt/esp/salinas1004.html

18. URQUIZO, Ángel (2004) Diseño de Proyectos de Graduación 